\title{
Global Texture Analysis of Iris Images for Ethnic Classification
}

\author{
Xianchao Qiu, Zhenan Sun, and Tieniu Tan \\ Center for Biometrics and Security Research, \\ National Laboratory of Pattern Recognition, Institute of Automation, \\ Chinese Academy of Sciences, P.O. Box 2728, Beijing, P.R. China, 100080 \\ \{xcqiu, znsun, tnt\}@nlpr.ia.ac.cn
}

\begin{abstract}
Iris pattern is commonly regarded as a kind of phenotypic feature without relation to the genes. In this paper, we propose a novel ethnic classification method based on the global texture information of iris images. So we would argue that iris texture is race related, and its genetic information is illustrated in coarse scale texture features, rather than preserved in the minute local features of state-of-the-art iris recognition algorithms. In our scheme, a bank of multichannel 2D Gabor filters is used to capture the global texture information and AdaBoost is used to learn a discriminant classification principle from the pool of the candidate feature set. Finally iris images are grouped into two race categories, Asian and non-Asian. Based on the proposed method, we get an encouraging correct classification rate (CCR) of $85.95 \%$ on a mixed database containing 3982 iris samples in our experiments.
\end{abstract}

\section{Introduction}

Iris texture is a distinct and stable biometric trait for personal identification. Some examples are shown in Fig. 1, which are from three different iris databases: CASIA [1] version 2, UPOL[2], and UBIRIS 3]. The iris of human eye is the annular part between the black pupil and the white sclera, in which texture is extremely rich.

Since Daugman's 4 iris recognition algorithm, many studies have been conducted on the randomness and uniqueness of human iris texture. Many people regard iris texture as phenotypic feature [4, 5, 6]. That is to say, the iris texture is the result of the developmental process and is not dictated by genetics. Even the genetically identical irises, the right and left pair from any given person have different textural appearance.

However, through investigating a large number of iris images of different races, Asian and non-Asian, we found that these iris patterns have different characteristics on the overall statistical measurement of the iris texture. At small scale, the details of iris texture are not dictated by genetics, but at large scale, the overall statistical measurement of iris texture is correlated with genetics. Motivated by this assumption, we try to do ethnic classification based on iris texture. 

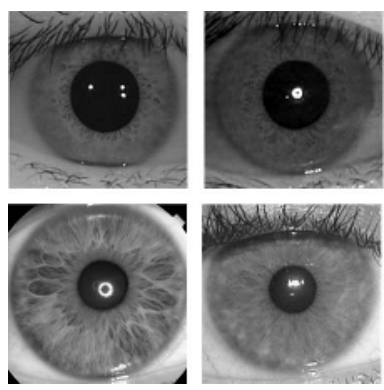

Fig. 1. Iris examples from different databases

So far, no work on ethnic classification with iris texture has been introduced in the public literature. In this paper, we propose a novel method for ethnic classification based on global texture analysis of iris images. Because the main purpose of this paper is to find the relationship between iris texture and race, only gray iris images are adopted in our experiments.

The remainder of this paper is organized as follows. Related work is presented in Section 2. The proposed method is discussed in Section 3. Experimental results are presented and discussed in Section 4 prior to conclusions in Section 5 .

\section{Related Work}

Ethnic classification is an old topic in social science. It is often assumed to be a fixed trait based on ancestry. But in natural science, few attempts have been made to perform automatic ethnic classification based on images of human. One example is Gutta et al. 7] with hybrid RBF/decision-trees. Using a similar architecture with Quinlan's C4.5 algorithm, they were able to achieve an average accuracy rate of $92 \%$ for ethnic classification based on face images. Recently, Shakhnarovich, Viola and Moghaddam [8] used a variant of AdaBoost to classify face images as Asian and non-Asian. Their approach yields a classifier which attains accuracy rate of $78 \%$. Lu and Jain 9 presented a Linear Discriminant Analysis (LDA) based scheme for two-class (Asian vs. nonAsian) ethnic classification from face images. Their reported accuracy is about $96 \%$.

\section{Global Texture Analysis}

In this paper, an ethnic classification algorithm includes three basic modules: image preprocessing, global feature extraction, and training. Fig. 2 shows how the proposed algorithm works. Detailed descriptions of these steps are as follows. 


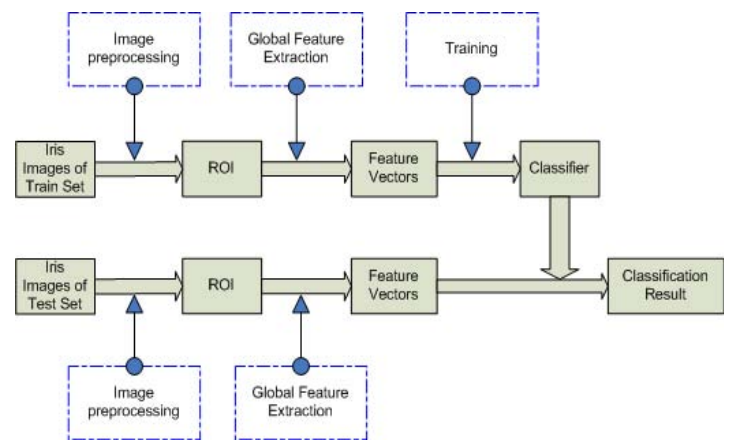

Fig. 2. The flowchart of our approach

\subsection{Image Preprocessing}

A typical iris recognition system must include image preprocessing. Fig. 3 illustrates the preprocessing step involving localization, normalization and enhancement. More details can be found in our previous work [6]. To exclude the eyelids and eyelashes, only the inner $3 / 4$ of the lower half of an iris region is used as the region of interest (ROI) for feature extraction, as shown in Fig. 3 (c). In our experiment, the size of ROI is $60 \times 256$ and it is divided into two equal regions, region $\mathrm{A}$ and region $\mathrm{B}$, as shown in Fig. 3 (d).

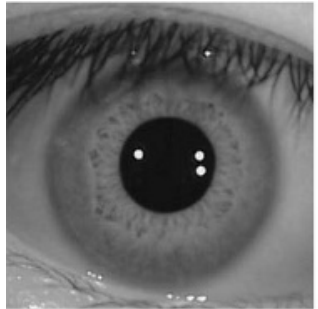

(a)

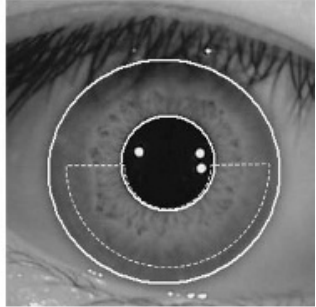

(b)

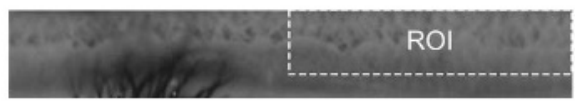

(c)

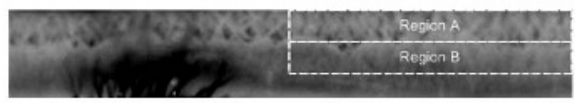

(d)

Fig. 3. Image preprocessing. (a) Original image. (b) Iris Localization. (c) Normalized image. (d) Normalized image after enhancement. 


\subsection{Global Feature Extraction}

Once ROI has been created, we can proceed with feature extraction based on multichannel Gabor filtering [10,11]. Gabor Energy 12] of each image point is used to represent texture features.

An input image (ROI) $I(x, y),(x, y) \in \Omega(\Omega$ denotes the set of image points ), is convolved with a $2 \mathrm{D}$ Gabor filter to obtain a Gabor filtered image $r(x, y)$.

$$
r(x, y)=\iint I\left(x_{1}, y_{1}\right) h_{i}\left(x-x_{1}, y-y_{1}\right) d x_{1} d y_{1} ; i=e, o .
$$

where $h_{e}$ and $h_{0}$ denote the even- and odd- symmetric Gabor filter.

The outputs of the even- and odd- symmetric Gabor filter in each image point can be combined into a single quantity called the Gabor energy 12 . This feature is defined as follows:

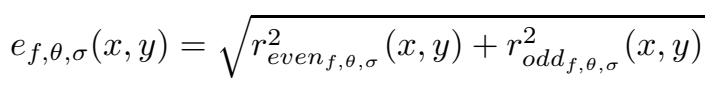

where $r_{\text {even }_{f, \theta, \sigma}}(x, y)$ and $r_{o d d_{f, \theta, \sigma}}(x, y)$ are the responses of even- and odd- symmetric Gabor filter respectively.

For Asians, region A has rich texture, but region B often has less texture. However for non-Asians, region A and region B nearly have the same rich texture. Thus, high-pass filtering could extract the discrimination between different races.

We design a bank of Gabor filters to extract Gabor energy features. Since the Gabor filters we use are of central symmetry in the frequency domain, only half of the frequency plan is need. Four values of orientation $\theta$ are used: $0, \frac{\pi}{4}, \frac{\pi}{2}$, and $\frac{3 \pi}{4}$. Because we are interested in the higher spatial frequencies in the frequency domain, for each orientation, we choose six spatial frequencies and ten space constants as follows:

$$
\begin{gathered}
f=0.25+2^{(i-0.5)} / 256, i=1,2, \ldots, 6 . \\
\sigma=3+i * 0.25, i=0,1, \ldots, 9 .
\end{gathered}
$$

It gives a total of 240 pairs of Gabor channels (four orientations, six frequencies combined with ten space constants).

For each pair of Gabor filters, we can get the Gabor energy image by Eqn. 2. Then the average Gabor energy values of region $\mathrm{A}$ and region $\mathrm{B}, m_{A}$ and $m_{B}$, are calculated.

In order to characterize global texture information of the ROI, two statistical features of the Gabor energy image, Gabor Energy (GE) and Gabor Energy Ratio (GER), are extracted.

$$
G E=m_{B}, \quad G E R=\frac{m_{A}}{m_{B}} .
$$

These features are combined to form the pool of candidate classifiers. 


\subsection{Training}

Many features have been extracted for each iris image, but our final application requires a very aggressive process which would discard the vast majority of features. For the sake of automatic feature selection, the AdaBoost algorithm 8 ] is used in our experiment to train the classifier.

\section{Experimental Results}

\subsection{Image Database}

Three iris databases are used in our experiments to evaluate the performance of the proposed method. They are CASIA[1, UPOL[2] and UBIRIS [3] iris image databases.

Because the iris images of the CASIA database are all from Asians in this version and the images of the UPOL and UBIRIS databases are mainly from Europeans, we divide all images into two categories, the Asian and the nonAsian. The Asian set includes 2400 images (all images from CASIA database), and the non-Asian set includes 1582 images (384 images from UPOL database and 1198 images from session-1 of UBIRIS database except 16 images without iris). All images from UPOL database and UBIRIS database are converted into 8 bit depth gray images as those in CASIA database. Then the images are separated into two sets: a training set of 1200 images (600 images randomly selected from the Asian and 600 images randomly selected from the non-Asian) and a testing set of 2782 images (the remaining images).

\subsection{Performance Evaluation of the Proposed Algorithm}

Statistical test is carried out to measure the accuracy of the algorithm. Correct Classification Rate (CCR) of the algorithm is examined.

Fig. 4 shows the distribution of Gabor Energy on the training set. The parameters of the Gabor filters used in this test were carefully selected to get the best peformance. When $f=0.338, \theta=\frac{\pi}{4}, \sigma=4$ and the threshold is set to 600 , the value of CCR is $77.92 \%$.

Fig. [5 shows the distribution of Gabor Energy Ratio on the training set. The parameters of the Gabor filters used in this test were $f=0.427, \theta=\frac{\pi}{4}, \sigma=4.5$ and the threshold is set to 0.93 . The value of CCR is $83.75 \%$.

For the sake of automatic feature selection, the AdaBoost algorithm was used in our experiment to learn a classification function. Given different feature sets, we get different results of classification, as shown in Table 1 .

From Table 1. we can draw a conclusion that Gabor Energy Ratio is better than Gabor Energy in representing texture features. But the highest Correct Classification Rate (CCR) is achieved when both Gabor Energy and Gabor Energy Ratio are used.

As mentioned before,Shakhnarovich et al. get a Correct Classification Rate (CCR) of $79.2 \%$ with 3500 images of human faces collected from the World Wide 


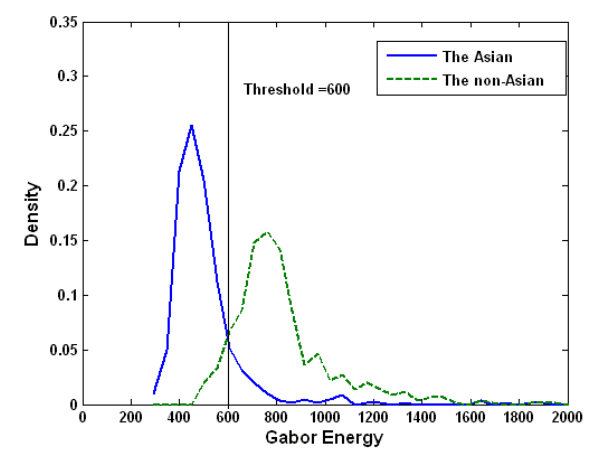

Fig. 4. Distribution of GE $(f=0.338$, $\left.\theta=\frac{\pi}{4}, \sigma=4\right)$

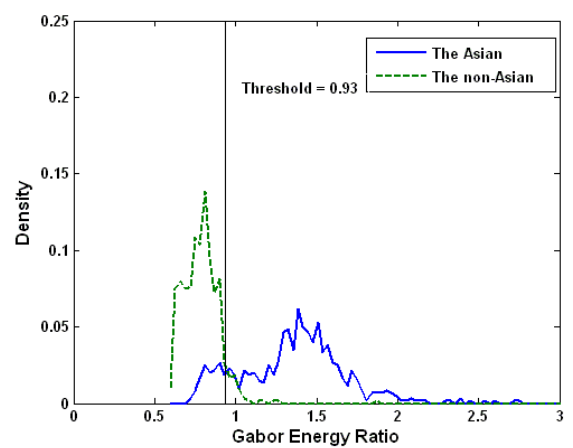

Fig. 5. Distribution of GER $(f=$ $\left.0.427, \theta=\frac{\pi}{4}, \sigma=4.5\right)$

Web. From the ethnic classification point of view, our method gets higher CCR of $85.95 \%$ than theirs.

Most of classification errors in our experiments are caused by three factors. Firstly, UBIRIS is a noisy iris image database and it includes many defocused images, which lacked of higher spatial frequencies. Secondly, the occlusions of eyelids and eyelashes in ROI may affect the classification result. Thirdly, there are some outliers in both classes. For example, an iris image from the Asian (CASIA database) may have very high Gabor energy in region B, while an iris image from the non-Asian (UPOL and UBIRIS database) may have very low Gabor energy in region B.

Images used in our experiments are acquired in different illumination. The UPOL database and UBIRIS database were acquired using visible light(VL) illumination, and CASIA database acquired in near infrared (NIR) illumination. In order to measure the influence of illumination conditions on the classification result, we conduct another experiment on a relatively small database. This database contains 480 iris images, 240 images are randomly selected from the CASIA database, and the other 240 images of 12 subjects were acquired using the same cameras but in visible light(VL) illumination, and all 480 images were from the Asian. All images are taken as the illumination testing set, it was divided into two classes, the VL and the NIR. Then three classifiers we had trained before were used for classification.

Table 1. Correct Classification Rate(CCR) resulted from the proposed method

\begin{tabular}{|c|c|c|c|c|c|}
\hline Feature Type & Number of & \multirow{2}{*}{$\begin{array}{c}\text { Number of } \\
\text { features }\end{array}$} & Colected features & Correct Classification Rate(\%) \\
\cline { 4 - 6 } & 240 & 4 & Training Set & Testing Set & Overall \\
\hline GE & 240 & 6 & 84.17 & 78.52 & 79.44 \\
\hline GER & 480 & 6 & 85.42 & 86.43 & 84.95 \\
\hline GE\&GER & 48.48 & 85.95 \\
\hline
\end{tabular}


Table 2. Correct Classification Rate on the illumination testing set

\begin{tabular}{|c|c|c|c|}
\hline $\begin{array}{c}\text { Feature } \\
\text { Type }\end{array}$ & $\begin{array}{c}\text { Number of } \\
\text { features }\end{array}$ & $\begin{array}{c}\text { Number of } \\
\text { selected features }\end{array}$ & $\begin{array}{c}\text { CCR on Illumination } \\
\text { Testing Set }(\%)\end{array}$ \\
\hline GE & 240 & 4 & 57.50 \\
\hline GER & 240 & 6 & 53.62 \\
\hline GE\&GER & 480 & 6 & 49.17 \\
\hline
\end{tabular}

As Table 2 shows, the classification result is only a little better than random guess as there are only two classes. The result demonstrates that the classifiers we had trained before were not tuned to classify the iris images in different illumination. The difference between iris images from different races is due to the inherent characteristics of iris texture.

\section{Conclusion}

In this paper, we have presented a novel method for automatic ethnic classification based on global texture analysis of iris images. A bank of multichannel 2D Gabor filters is used to capture the global texture information in some iris regions. An AdaBoost learning algorithm is used to select the features and train the classifier. Using the proposed method, we get an encouraging correct classification rate (CCR) of $85.95 \%$ in our experiments. Based on the analytical and experimental investigations presented in this paper, the following conclusion may be drawn: 1)At a small scale, the local features of the iris are unique to each subject, whereas at a large scale, the global features of the iris are similar for a specific race, and they seem to be dependent on the genes; 2)and the global texture features of iris are efficient for ethnic classification.

\section{Acknowledgement}

This work is funded by research grants from the National Basic Research Program (Grant No. 2004CB318110), the Natural Science Foundation of China (Grant No. 60335010, 60121302, 60275003, 60332010, 69825105) and the Chinese Academy of Sciences.

\section{References}

1. Chinese Academy of Sciences Institute of Automation. CASIA iris image database, http://www.sinobiometrics.com. 2003.

2. Michal Dobes and Libor Machala. Upol iris image database, http://phoenix.inf.upol.cz/iris/. 2004.

3. Hugo Proenca and Luis A. Alexandre. Ubiris iris image database, http://iris.di.ubi.pt. 2004.

4. John Daugman. High confidence visual recognition of persons by a test of statistical independence. IEEE TRANS. PAMI, 15(11):1148-1161, 1993. 
5. R.P. Wildes. Iris recognition: An emerging biometric technology. Proceedings of the IEEE, 85(9):1348-1363, 1997.

6. Li Ma, Tieniu Tan, Yunhong Wang, and Dexin Zhang. Personal identification based on iris texture analysis. IEEE TRANS. PAMI, 25(12):1519-1533, 2003.

7. S. Gutta, H. Wechsler, and P. J. Phillips. Gender and ethnic classification. In International Conference on Automatic Face and Gesture Reconition, pages 194199, 1998.

8. Gregory Shakhnarovich, Paul A. Viola, and Baback Moghaddam. A unified learning framework for real time face detection and classification. In International Conference on Automatic Face and Gesture Reconition, 2002.

9. Xiaoguang Lu and Anil K. Jain. Ethnicity identification from face images. In Proc. SPIE Defense and Security Symposium, April 2004.

10. Yong Zhu, Tieniu Tan, and Yunhong Wang. Font recognition based on global texture analysis. IEEE TRANS. PAMI, 23(10):1192-1200, 2001.

11. Tieniu Tan. Rotation invariant texture features and their use in automatic script indentification. IEEE TRANS. PAMI, 20(7):751-756, 1998.

12. Simona E. Grigorescu, Nicolai Petkov, and Peter Kruizinga. Comparison of texture features based on gabor filters. IEEE Transactions on Image Processing, 11(10):1160-1167, 2002. 